\title{
Virulotyping of Salmonella enterica subsp. enterica isolated from indigenous vegetables and poultry meat in Malaysia using multiplex-PCR.
}

\begin{abstract}
The increased occurrence of Salmonella occurrence in local indigenous vegetables and poultry meat can be a potential health hazards. This study is aimed to detect the prevalence of twenty different virulence factors among Salmonella enterica strains isolated from poultry and local indigenous vegetables in Malaysia via an optimized, rapid and specific multiplex PCR assay. The assay encompasses a total of 19 Salmonella pathogenicity islands genes and a quorum sensing gene (sdiA) in three multiplex reaction sets. A total of 114 Salmonella enterica isolates belonging to 38 different serovars were tested. Each isolate in under this study was found to possess up to $70 \%$ of the virulence genes tested and exhibited variable pathogenicity gene patterns. Reproducibility of the multiplex PCR assay was found to be $100 \%$ and the detection limit of the optimized multiplex PCR was tested with lowest detectable concentration of DNA $0.8 \mathrm{pg}$ ?1-1. This study demonstrated various Salmonella pathogenicity island virulence gene patterns even within the same serovar. This sets of multiplex PCR system provide a fast and reliable typing approach based on Salmonella pathogenicity islands, thus enabling an effective monitoring of emerging pathogenic Salmonella strains as an additional tool in Salmonella surveillance studies.
\end{abstract}

Keyword: Virulotyping; Salmonella enterica; Salmonella pathogenicity islands (SPIs); Multiplex PCR. 